Classification

Physics Abstracts

$61.50 \mathrm{~J}-61.70 \mathrm{~N}$

\title{
Shear modulations and icosahedral twins
}

\author{
Michel Duneau \\ Centre de Physique Théorique, Ecole Polytechnique, 91128 Palaiseau Cedex, France \\ (Received October 01, 1990; accepted January 15, 1991)
}

\begin{abstract}
Résumé. - Nous montrons que si deux réseaux $\mathrm{L}_{\mathrm{a}}$ et $\mathrm{L}_{b}$ sont liés par un cisaillement, il existe une transformation displacive simple, correspondant à une modulation périodique de $\mathrm{L}_{\mathbf{a}}$, qui transforme $\mathrm{L}_{\mathbf{a}}$ en $\mathrm{L}_{\mathbf{b}}$. Nous considérons les maclages icosaèdriques récemment observés dans les alliages AlFeMnSi et montrons qu'ils sont liés par des rotations équivalentes au produit de seulement deux tels cisaillements.
\end{abstract}

\begin{abstract}
We show that given two lattices $\mathrm{L}_{\mathbf{a}}$ and $\mathrm{L}_{\mathbf{b}}$, related be a shear transformation, there exists a simple displacive transformation, corresponding to a periodic modulation of $\mathrm{L}_{\mathbf{a}}$, which transforms $\mathrm{L}_{\mathbf{a}}$ into $\mathrm{L}_{\mathbf{b}}$. We consider recently observed icosahedral twinnings in AlFeMnSi alloys and show that they are related by rotations equivalent to the product of only two such shear transformations.
\end{abstract}

\section{Introduction.}

Shear transformations are in some the most simple transformations between different lattices. From a geometrical point of a view they are characterized by a direction, an invariant plane and a magnitude. Only in specific situations, such as the martinsitic transformation, do these transformations correspond to real displacements of atoms. In other situations, they are involved in purely geometrical relations between two lattices related by a transition, whereas the actual transformation is believed to be displacive. This is the case of the "second shear" in the martinsitic transformation.

In this paper we show that if two lattices are related by a macroscopic linear shear transformation, a simple displacive transformation between these lattices can be devised. This bounded transformation involves a periodic displacement field of small amplitude in such a way that the second lattice turns out to be a modulation of the first one.

Such a situation is however restricted to particular pairs of lattices. Generally, two lattices of equal density are related by the combination of 4 successive shear transformations. In this case a displacive transformation can still be performed but the corresponding modulation is far more complex and the displacement field is usually quasiperiodic. 
We don't claim that actual displacements are exactly those given those by our construction. Relaxation processes normally would change the trajectories of atoms. But the proposed solutions for displacive transformations are in some sense the most simple and could be associated to simple soft modes.

Even if a structural transformation is not observed, the relative orientations of grains in a polycrystalline system can be analysed along the same lines.

In the case of twinnings, a generic rotation similarly corresponds to 4 different shears. Of specific interest are the rotations for which only two shear transformations monitor an equivalent displacive transformation. In other words, particular orientational relationships are pointed out by a requirement of simplicity of an actual or potential transformation. In this respect we show that the recently observed icosahedral twins in AlFeMnSi alloys are associated to simple displacive transformations observed icosahedral twins in AlFeMnSi alloys are associated to simple displacive transformations involving only two successive simple modulations.

\section{Shear transformation and shear modulation.}

In this section we show that shear transformations, which are linear and therefore macroscopic transformations, have simple local and displacive analogs called hereafter shear modulations.

Let $\mathrm{L}_{\mathrm{a}}=A \mathrm{Z}^{3}$ and $\mathrm{L}_{\mathbf{b}}=B \mathrm{Z}^{3}$ denote two lattices with respective basis $\left\{\mathbf{a}_{1}, \mathbf{a}_{2}, \mathbf{a}_{3}\right\}$ and $\left\{\mathbf{b}_{1}\right.$, $\left.\mathbf{b}_{2}, \mathbf{b}_{3}\right\}$ given by $\mathbf{a}_{i}=A \mathbf{e}_{i}$ and $\mathbf{b}_{i}=B \mathbf{e}_{i}(i=1,2,3)$ where $\left\{\mathbf{e}_{1}, \mathbf{e}_{2}, \mathbf{e}_{3}\right\}$ is the standard basis of the 3- dimensional space. $A$ and $B$ are $3 \times 3$ matrices with non vanishing determinants and if $\mathrm{L}_{\mathrm{a}}$ and $\mathrm{L}_{\mathrm{b}}$ have the same density we have $\operatorname{det}(A)= \pm \operatorname{det}(B)$.

Actually, the matrices $A$ and $B$ are not uniquely defined by the lattices $\mathrm{L}_{\mathrm{a}}$ and $\mathrm{L}_{\mathbf{b}}$ and similarly, the $\mathbf{a}$-basis and $\mathbf{b}$-basis are not unique. All other equivalent description involve modular matrices $U$ and $V$, i.e. with integer entries and determinant equal to \pm 1 , in such a way that $\mathrm{L}_{\mathbf{a}}=A U \mathrm{Z}^{3}$ and $\mathrm{L}_{\mathrm{b}}=B V \mathrm{Z}^{3}$. The corresponding basis are respectively $\left\{\alpha_{1}, \alpha_{2}, \alpha_{3}\right\}$, given by $\alpha_{i}=A U \mathbf{e}_{i}$, and $\left\{\beta_{1}, \beta_{2}, \beta_{3}\right\}$, given by $\beta_{i}=B V \mathbf{e}_{i}(i=1,2,3)$.

We shall first consider pairs of lattices $\mathrm{L}_{\mathrm{a}}$ and $\mathrm{L}_{\mathrm{b}}$ related by simple shear transformations. By this we mean that $\mathrm{L}_{b}=\mathcal{S} \mathrm{L}_{\mathrm{a}}$ where the linear transformation $\mathcal{S}$ has the following property: $\mathrm{L}_{\mathrm{a}}$ and $\mathrm{L}_{\mathrm{b}}$ have respective basis $\left\{\alpha_{1}, \alpha_{2}, \alpha_{3}\right\}$ and $\left\{\beta_{1}, \beta_{2}, \beta_{3}\right\}$ such that

$$
\begin{aligned}
& \beta_{1}=\mathcal{S}\left(\alpha_{1}\right)=\alpha_{1}+s_{1} \alpha_{3} \\
& \beta_{2}=\mathcal{S}\left(\alpha_{2}\right)=\alpha_{2}+s_{2} \alpha_{3} \\
& \beta_{3}=\mathcal{S}\left(\alpha_{3}\right)=\alpha_{3}
\end{aligned}
$$

where $s_{1}$ and $s_{2}$ are two parameters giving the amplitude of the shear transformation, the direction of which is given by the lattice direction $\alpha_{3}=\beta_{3}$.

These particular basis are associated, as mentioned above, to particular modular matrices $U$ and $V$ and it follows from (2.1) that

$$
\beta_{i}=B V \mathbf{e}_{i}=A U S \mathbf{e}_{i} \quad \text { with } \quad S=\left(\begin{array}{ccc}
1 & 0 & 0 \\
0 & 1 & 0 \\
s_{1} & s_{2} & 1
\end{array}\right)
$$

Therefore we have $B V=A U S$ and consequently the transition matrix between $\mathrm{L}_{\mathbf{a}}$ and $\mathrm{L}_{\mathbf{b}}$ reads

$$
T=A^{-1} B=U S V^{-1}
$$


Such a shear transformation obviously preserves density since det $(S)=1$. The shear transformation has an invariant plane $\mathrm{P}$ such that $\mathcal{S P}=\mathrm{P}$ which is generated by $\alpha_{3}$ and $s_{2} \alpha_{1}-s_{1} \alpha_{2}$. P is not in general a lattice plane of $\mathrm{L}_{\mathrm{a}}$, but if $s_{1}$ and $s_{2}$ are both rational numbers.

Any lattice point $x$ of $\mathrm{L}_{\mathrm{a}}$ reads $x=\Sigma x_{i} \alpha_{i}=A U X$, where $X$ is a column vector $X$ with integer entries $x_{1}, x_{2}$ and $x_{3}$. In view of (2.1) the mapping $\mathcal{S}$ transforms a lattice point $x=\Sigma x_{i} \alpha_{i}$ of $L_{a}$ into the lattice point $y=\Sigma x_{i} \beta_{i}=B V X$ of $\mathrm{L}_{\mathrm{b}}$. The coordinates $Y$ of $y$ in the $\alpha$-basis satisfy $y=A U Y$ from which we easily derive

$$
Y=S X
$$

Obvioulsy the distance between $x$ and $y=\mathcal{S}(x)$ is not bounded since

$$
y-x=x_{1}\left(\beta_{1}-\alpha_{1}\right)+x_{2}\left(\beta_{2}-\alpha_{2}\right)=\left(x_{1} s_{1}+x_{2} s_{2}\right) \alpha_{3}
$$

Now, assuming that $\mathrm{L}_{\mathrm{a}}$ and $\mathrm{L}_{\mathrm{b}}$ are related by a such a simple shear transformation, we show that a simple transformation from $\mathrm{L}_{a}$ to $\mathrm{L}_{b}$ can be performed by means of bounded displacements: this means that there exists a global mapping $\Sigma$ from $\mathrm{L}_{\mathrm{a}}$ to $\mathrm{L}_{\mathbf{b}}$, that well call shear modulation for obvious reasons, such that for all $x$ in $\mathrm{L}$ the distance between $x$ and $z=\Sigma(x)$ in $\mathrm{L}_{\mathrm{b}}$ remains bounded. Any lattice point $x=\Sigma x_{i} \alpha_{i}$ of $\mathrm{L}_{\mathrm{a}}$ can be decomposed in the $\beta$-basis:

$$
x=x_{1}\left(\beta_{1}-s_{1} \beta_{3}\right)+x_{2}\left(\beta_{2}-s_{2} \beta_{3}\right)+x_{3} \beta_{3}=y_{1} \beta_{1}+y_{2} \beta_{2}+y_{2} \beta_{3}
$$

with

$$
\begin{aligned}
& y_{1}=x_{1} \\
& y_{2}=x_{2} \\
& y_{3}=x_{3}-s_{1} x_{1}-s_{2} x_{2}
\end{aligned}
$$

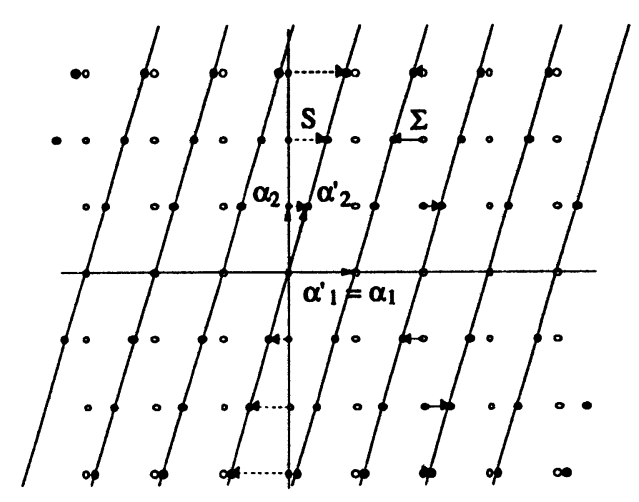

Fig. 1. - The white lattice $\mathrm{L}$, generated by $\left\{\alpha_{1}, \alpha_{2}\right\}$, is transformed into $\mathrm{L}^{\prime}$ generated by $\left\{\alpha_{1}^{\prime}=\alpha_{1}, \alpha_{2}^{\prime}=\right.$ $\left.\alpha_{2}+s \alpha_{1}\right\}$. The shear transformation $S$, represented by dotted arrows is not bounded, while the shear modulation $\Sigma$, represented by black arrows, is bounded.

So the third coordinate $y_{3}$ is not, in general, an integer (since $x$ is not a lattice point of $\mathrm{L}_{b}$ ). If $\operatorname{Rnd}(x)$ denotes the round function, i.e. $\operatorname{Rnd}(x)=n$ if $n-1 / 2<x \leq n+1 / 2$, then the point 
defined by $z=x_{1} \beta_{1}+x_{2} \beta_{2}+\operatorname{Rnd}\left(x_{3}-s_{1} x_{1}-s_{2} x_{2}\right) \beta_{3}$ belongs to the lattice $\mathrm{L}_{\mathrm{b}}$. Furthermore the difference between $x$ and $z=\Sigma(x)$ is given by

$$
z-x=-\operatorname{Frac}\left(x_{3}-s_{1} x_{1}-s_{2} x_{2}\right) \alpha_{3}
$$

Where $\operatorname{Frac}(x)=x-\operatorname{Rnd}(x)$ is a function with value between $-1 / 2$ and $1 / 2$.

So the distance between $x$ and $z$ is always bounded by $\left\|\alpha_{3}\right\| / 2=\left\|\beta_{3}\right\| / 2$ (see Fig. 1).

It was proved in [2] that the shear modulation $\Sigma: x \rightarrow z$ from $\mathrm{L}_{\mathrm{a}}$ to $\mathrm{L}_{\mathrm{b}}$ is actually one-to-one and can be extended to a periodic piecewise linear mapping on the whole physical space. $\Sigma$ differs from the identiy mapping by a bounded displacement $d$ :

$$
\begin{gathered}
\Sigma(x)=x+d(x) \\
d(x)=- \text { Frac }\left(x_{3}-s_{1} x_{1}-s_{2} x_{2}\right) \alpha_{3}
\end{gathered}
$$

where $\left\{x_{1}, x_{2}, x_{3}\right\}$ are the coordinates of $x$ in the $\alpha$-basis of $\mathrm{L}_{\mathrm{a}}$.

Since the Frac function is periodic with period $1, \operatorname{Frac}(x+n)=\operatorname{Frac}(x)$, the displacement field $d(x)$ is a periodic modulation field which reads $d(x)=\operatorname{Frac}(q \cdot x) \alpha_{3}$ for some $q$ in the reciprocal space. Finally, $\mathrm{L}_{\mathbf{b}}$ can be considered as a periodic modulation of $\mathrm{L}_{\mathbf{a}}$, although the period of $d$ is generally incommensurate with $\mathrm{L}_{\mathbf{a}}$.

Now let $\mathrm{L}_{\mathrm{a}}=A \mathrm{Z}^{3}$ and $\mathrm{L}_{\mathrm{b}}=B \mathrm{Z}^{3}$ be any two lattices with equal density (det $A= \pm \operatorname{det} B$ ). The linear transformation $B A^{-1}$ from $\mathrm{L}_{\mathrm{a}}$ to $\mathrm{L}_{\mathbf{b}}$ is represented by the matrix $T=A^{-1} B$ in the natural basis of $\mathrm{L}_{\mathrm{a}}$ and det $T= \pm 1$.

In general, these lattices will not be related by a unique displacive transformation as above, even with the free choice of the modular matrices $U$ and $V$ : there are not enough continuous parameters. But the transition matrices $T$ can be decomposed into a product of shear transformations. More precisely, it is proved in [2] that there exists a modular matrix $U$, with integer entries and determinant \pm 1 , and at most 4 shear transformations $S_{0}, S_{1}, S_{2}$ and $S_{3}$ such that

$$
T=A^{-1} B=U S_{0} S_{3} S_{2} S_{1}
$$

(compare to (2.3)) where

$$
\begin{aligned}
S_{0} & =\left(\begin{array}{ccc}
1 & s_{02} & s_{03} \\
0 & 1 & 0 \\
0 & 0 & 1
\end{array}\right) S_{3}=\left(\begin{array}{ccc}
1 & 0 & 0 \\
0 & 1 & 0 \\
s_{31} & s_{32} & 1
\end{array}\right) S_{2}=\left(\begin{array}{ccc}
1 & 0 & 0 \\
s_{21} & 1 & s_{23} \\
0 & 0 & 1
\end{array}\right) \\
S_{1} & =\left(\begin{array}{ccc}
1 & s_{12} & s_{13} \\
0 & 1 & 0 \\
0 & 0 & 1
\end{array}\right)
\end{aligned}
$$

Usually, the modular matrix is the identity matrix but for particular transition matrices, a change of basis, performed by $U$, is necessary.

Once the transition matrix $T$ is decomposed into a product of shear transformations we can apply the above results to build a bounded one to one mapping $\Sigma$ between $\mathrm{L}_{\mathbf{a}}$ and $\mathrm{L}_{\mathrm{b}}$. This is achieved simply by replacing each linear shear transformation $\mathcal{S}_{i}$, associated to the matrix $S_{i}$, by the corresponding bounded shear modulation $\Sigma_{i}: \Sigma=\Sigma_{0} \Sigma_{3} \Sigma_{2} \Sigma_{1}$.

The resulting displacement field $d(x)$ is defined by $\Sigma(x)=x+d(x)$. However this modulation is far more complicated: in particular $d$ involves as many frequencies $\left\{q_{0}, q_{3}, q_{2}, q_{1}\right\}$ as shears transformations in the decomposition. As a consequence the modulation is generally quasiperiodic. 
The decomposition (2.11) can be obtained by an explicit algorithm. For simplicity, we only give here the 2-dimensional analog of this procedure: if $T$ is a $2 \times 2$ matrix with determinant \pm 1 , there exist a $2 \times 2$ modular matrix $U$ and at most 3 shear transformations $S_{0}, S_{1}$ and $S_{2}$ such that

$$
T=U S_{0} S_{2} S_{1}
$$

where $S_{0}=\left(\begin{array}{cc}1 & s_{0} \\ 0 & 1\end{array}\right), S_{2}=\left(\begin{array}{cc}1 & 0 \\ s_{2} & 1\end{array}\right), S_{1}=\left(\begin{array}{cc}1 & s_{1} \\ 0 & 1\end{array}\right)$

Actually the product of shear matrices reads

$$
S_{0} S_{2} S_{1}=\left(\begin{array}{ll}
1+s_{0} s_{2} & s_{0}+s_{1}\left(1+s_{0} s_{2}\right) \\
s_{2} & 1+s_{2} s_{1}
\end{array}\right)
$$

The three parameters $s_{0}, s_{1}$ and $s_{2}$ are easily derived from the entries of $T$. Notice that a modular matrix $U$, different from the identity, is needed if $T_{21}=0$; actually, if $U=I$ and $T_{21}=0$, we get $s_{2}=0$ which would suppose that $T_{11}=T_{22}=1$.

If $\mathrm{L}_{\mathrm{a}}$ and $\mathrm{L}_{\mathrm{b}}$ denote the two square lattices related by a $\pi / 4$ rotation, $T$ is simply the $\pi / 4$ rotation and the above decomposition reads

$$
T=1 / \sqrt{2}\left(\begin{array}{rr}
1 & -1 \\
1 & 1
\end{array}\right)=\left(\begin{array}{cc}
1 & \sqrt{2-1} \\
0 & 1
\end{array}\right)\left(\begin{array}{cc}
1 & 0 \\
-1 / \sqrt{2} & 1
\end{array}\right)\left(\begin{array}{cc}
1 & \sqrt{2-1} \\
0 & 1
\end{array}\right)
$$

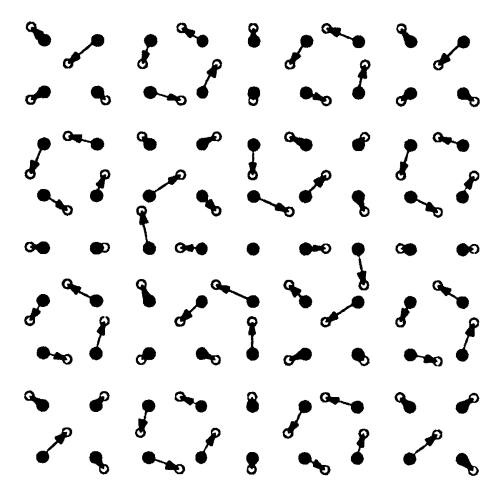

Fig. 2. - The quasiperiodic one to one mapping between the two square lattices at $\pi / 4$.

If $x=x_{1} \mathbf{a}_{1}+x_{2} \mathbf{a}_{2}$ is in $\mathrm{L}_{\mathbf{a}}$ and $y=y_{1} \mathbf{b}_{1}+y_{2} \mathbf{b}_{2}$ is in $\mathrm{L}_{\mathbf{b}}$ the corresponding bounded one to one mapping from $L_{a}$ to $L_{b}$ (see Fig. 2) is given by

$$
\begin{aligned}
y_{1}= & x_{1}+\operatorname{Rnd}\left[(\sqrt{2}-1) x_{2}\right]+ \\
& +\operatorname{Rnd}\left[(\sqrt{2}-1)\left(x_{2}+\operatorname{Rnd}\left(-\sqrt{2}\left(x_{1}+\operatorname{Rnd}\left((\sqrt{2}-1) x_{2}\right)\right) / 2\right)\right)\right] \\
y_{2}= & x_{2}+\operatorname{Rnd}\left[-\sqrt{2}\left(x_{1}+\operatorname{Rnd}\left((\sqrt{2}-1) x_{2}\right)\right) / 2\right]
\end{aligned}
$$

Remark. Such bounded one to one mappings between lattices are not obvious. Consider for instance the case of two square lattices $\mathrm{L}_{a}$ and $\mathrm{L}_{b}$ in the plane with a $\pi / 4$ relative orientation. A 
natural candidate for such a map consists in mapping a point $x$ of $\mathrm{L}_{\mathrm{a}}$ onto the origin $y$ of the square of $L_{b}$ which contains $x$. However, squares of $L_{b}$ may contain 0,1 or 2 points of $L_{a}$ so that the one to one property cannot be satisfied.

The number of shear transformations, 4 in $3 \mathrm{D}$ and 3 in $2 \mathrm{D}$, is a maximum. In $3 \mathrm{D}$ a transition matrix $T=A^{-1} B$ involves 8 independent continuous parameters because $\operatorname{det}[T]= \pm 1$. Since each shear transformation involves only 2 parameters, four of them must be combined to cover all cases.

But, for certain lattices and transition matrices the decomposition may involves less shear transformations. In view of the decomposition (2.11) these cases correspond to at least one shear transformation being equal to the identity.

This reduction is exceptional and we think that it may have an important physical meaning by specifying particular orientational relationships. For instance if $\mathrm{L}_{\mathrm{b}}=R \mathrm{~L}_{\mathrm{a}}$, where $R$ is a rotation, the transition matrix is $T=A^{-1} B=A^{-1} R A$ and the decomposition reads $A^{-1} R A=U S_{0} S_{3} S_{2}$ $S_{1}$. The point is that for exceptional rotations $R$, the decomposition may involve only 3 or even 2 shear transformations. This is actually observed in the case of icosahedral twinnings as we show in next section.

\section{Icosahedral twins.}

In this section we show that icosahedral twins of cubic lattices, i.e. twins related by a $2 \pi / 5$ rotation around a pseudo 5-fold axis of type $\langle 1 \tau 0\rangle(\tau$ is the golden mean $(1+\sqrt{5}) / 2)$, can be viewed as corresponding to a coincidence lattice, up to simple shear transformations (see also [3]).

Let $\mathbf{a}_{1}=(1,0)$ and $\mathbf{a}_{2}=(1 / 2, \sqrt{3} / 2)$ denote the generators of the $2 \mathrm{D}$-lattice L corresponding to the (111) -plane of a cubic lattice of convenient parameter. Then

$$
\mathrm{L}=A \mathrm{Z}^{2}
$$

where $A=\left(\begin{array}{cc}1 & 1 / 2 \\ 0 & \sqrt{3} / 2\end{array}\right)$ and $\mathrm{Z}^{2}$ is the standard square lattice.

Let $\Lambda_{\mathrm{a}}$ denote the sub-lattice of L spanned by $\alpha_{1}=2 \mathbf{a}_{1}+2 \mathbf{a}_{2}$ and $\alpha_{2}=\mathbf{a}_{2}$. Thus $\Lambda_{\mathrm{a}}$ is of index 2 in $\mathrm{L}$ and can be obtained as follows:

$$
\Lambda_{\mathrm{a}}=A M \mathrm{Z}^{2} \text { with } \quad M=\left(\begin{array}{ll}
2 & 0 \\
2 & 1
\end{array}\right)
$$

Now we consider a lattice $\Lambda_{\mathrm{a}}^{\prime}$ which is deduced from $\Lambda_{\mathrm{a}}$ by a shear transformation $S_{\mathrm{a}}$ which transforms the basis $\left\{\alpha_{1}, \alpha_{2}\right\}$ into $\left\{\alpha_{1}^{\prime}, \alpha_{2}^{\prime}\right\}: \alpha_{1}^{\prime}=\alpha_{1}$ and $\alpha_{2}^{\prime}=\frac{\tau-1}{2} \alpha_{1}+\alpha_{2}$. Consequently

$$
\Lambda_{\mathrm{a}}^{\prime}=A M S_{\mathrm{a}} \mathrm{Z}^{2} \text { with } S_{\mathrm{a}}=\left(\begin{array}{cc}
1 & (\tau-1) / 2 \\
0 & 1
\end{array}\right)
$$

Similarly let $\Lambda_{b}$ denote the sub-lattice of $L$ spanned by $\beta_{1}=4 \mathbf{a}_{1}-\mathbf{a}_{2}$ and $\beta_{2}=2 \mathbf{a}_{1}$. Thus $\Lambda_{b}$ is of index 2 in $L$ and can be obtained as follows:

$$
\Lambda_{\mathrm{b}}=A N Z^{2} \quad \text { with } \quad N=\left(\begin{array}{cc}
4 & 2 \\
-1 & 0
\end{array}\right)
$$

A lattice $\Lambda_{\mathrm{b}}^{\prime}$ is derived from $\Lambda_{\mathrm{b}}$ by a shear transformation $S_{\mathrm{b}}$ which transforms the basis $\left\{\beta_{1}, \beta_{2}\right\}$ into $\left\{\beta_{1}^{\prime}, \beta_{2}^{\prime}\right\}: \beta_{1}^{\prime}=\beta_{1}+\frac{3 r-5}{2} \beta_{2}$ and $\beta_{2}^{\prime}=\beta_{2}$. Therefore

$$
\Lambda_{\mathrm{b}}^{\prime}=A N S_{\mathrm{b}} \mathrm{Z}^{2} \quad \text { where } \quad S_{\mathrm{b}}=\left(\begin{array}{cc}
1 & 0 \\
(3 \tau-5) / 2 & 1
\end{array}\right)
$$


Notice that $\Lambda_{\mathrm{a}}^{\prime}$ and $\Lambda_{\mathrm{b}}^{\prime}$ have equal density since $\operatorname{det}(M)=\operatorname{det}(N)=2$.

The point is that $\Lambda_{\mathrm{b}}^{\prime}$ can be deduced from $\Lambda_{\mathrm{a}}^{\prime}$ by a mere rotation; this can be checked by computing the following norms and scalar products: $\left|\alpha_{1}^{\prime}\right|=\left|\beta_{1}^{\prime}\right|=2 \sqrt{3},\left|\alpha_{2}^{\prime}\right|=\left|\beta_{2}^{\prime}\right|=2$ and $\left(\alpha_{1}^{\prime}, \alpha_{2}^{\prime}\right)=$ $\left(\beta_{1}^{\prime}, \beta_{2}^{\prime}\right)=6 \tau-3$. More precisely if follows from the above definition that $\Lambda_{\mathbf{b}}^{\prime}=A N\left(S_{\mathbf{b}} S_{\mathbf{a}}^{-1}\right) M^{-1}$ $A^{-1} \Lambda_{\mathrm{a}}^{\prime}$. A simple computation gives the transition matrix

$$
A N\left(S_{\mathrm{b}} S_{\mathrm{a}}^{-1}\right) M^{-1} A^{-1}=\left(\begin{array}{cc}
\frac{3 \tau-2}{4} & \frac{\tau \sqrt{3}}{4} \\
-\frac{\tau \sqrt{3}}{4} & \frac{3 \tau-2}{4}
\end{array}\right)
$$

which is a rotation $r$ of angle $\operatorname{Arccos}((3 \tau-2) / 4)=\operatorname{Arcsin}(\tau \sqrt{3} / 4) \approx 44.48^{\circ}$.

Such orientation relations between grains of cubic systems have been observed in rapidly quenched alloys of $\mathrm{Al}_{75} \mathrm{Mn}_{15-x} \mathrm{Fe}_{x} \mathrm{Si}_{10}$ (see [1]) with $x=5$ or 10. As pointed out in [1], this $44.48^{\circ}$ rotation around an [111] axis of a cubic lattice is equivalent to a $2 \pi / 5$ rotation around the pseudo "five-fold axis" $<1 \tau 0>$. More precisely, a five-fold rotation around [1 $\tau 0]$ generates five different variants of the cubic system. Any two of them share a three-fold axis and they are related by the above $44.48^{\circ}$ rotation.

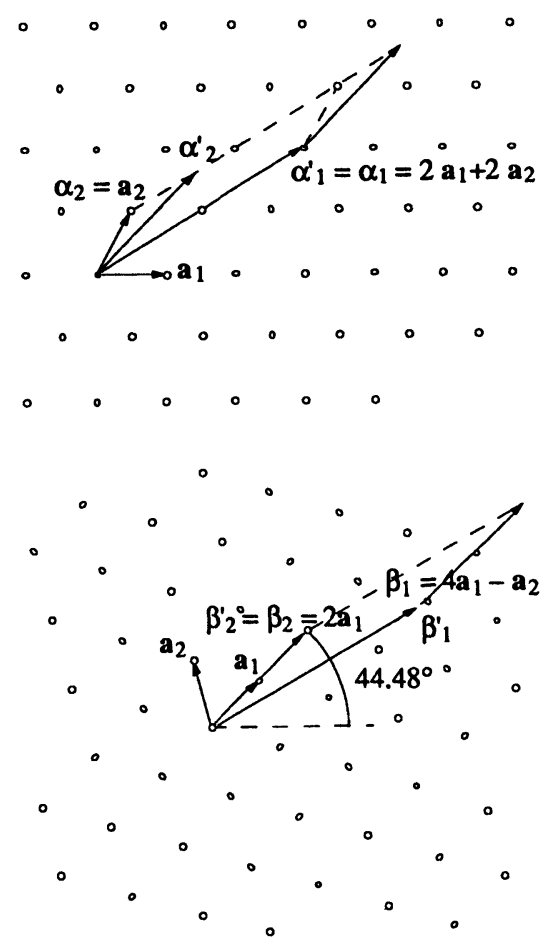

Fig. 3. - Notice that since $5 / 3$ is a good approximant of $\tau, \beta_{1}^{\prime}=r \alpha_{1}$ is very close to $\beta_{1}=4 \mathbf{a}_{1}-\mathbf{a}_{2}$ and therefore $\Lambda_{\mathbf{b}}^{\prime} 1 \mathrm{~s}$, in some sense, almost a sub-lattice of $\mathrm{L}$. 
Acknowledgements.

Part of this work is closely related with previous works with C. Oguey and A. Thalal. I am indebted to them for many helpful and constructive discussions.

\section{References}

[1] BENDERSKi L.A., CAHN J.W., Gratias D., Phil. Mag. 60 (1989) 837.

[2] Duneau M., Oguey C., J. Phys. A 24 (1991) 461.

[3] DUNEAU M., OGUEY C., Europhys. Lett. 13 (1990) 67. 